\title{
Sensitization of Transient Receptor Potential Vanilloid 1 by the Prokineticin Receptor Agonist Bv8
}

\author{
Vittorio Vellani, ${ }^{1,2}$ Mariantonella Colucci, ${ }^{1,3}$ Roberta Lattanzi, ${ }^{3}$ Elisa Giannini, ${ }^{3}$ Lucia Negri, ${ }^{3}$ Pietro Melchiorri, ${ }^{3}$ and \\ Peter A. McNaughton ${ }^{1}$ \\ ${ }^{1}$ Department of Pharmacology, University of Cambridge, Cambridge CB2 1PD, United Kingdom, ${ }^{2 D i p a r t i m e n t o ~ d i ~ S c i e n z e ~ B i o m e d i c h e, ~ v i a ~ C a m p i ~ 287, ~}$ \\ Università degli Studi di Modena e Reggio Emilia, I-41100 Modena, Italy, and ${ }^{3}$ Dipartimento di Fisiologia Umana e Farmacologia "Vittorio Erspamer," \\ Università di Roma “La Sapienza," 00185 Roma, Italy
}

\begin{abstract}
Small mammalian proteins called the prokineticins [prokineticin 1 (PK1) and PK2] and two corresponding G-protein-coupled receptors [prokineticin receptor 1 (PKR1) and PKR2] have been identified recently, but the physiological role of the PK/PKR system remains mostly unexplored. Bv8, a protein extracted from frog skin, is a convenient and potent agonist for both PKR1 and PKR2, and injection of Bv8 in vivo causes a potent and long-lasting hyperalgesia. Here, we investigate the cellular basis of hyperalgesia caused by activation of PKRs. Bv8 caused increases in $[\mathrm{Ca}]_{\mathrm{i}}$ in a population of isolated dorsal root ganglion (DRG) neurons, which we identified as nociceptors, or sensors for painful stimuli, from their responses to capsaicin, bradykinin, mustard oil, or proteases. Bv8 enhanced the inward current carried by the heat and capsaicin receptor, transient receptor potential vanilloid 1 (TRPV1) via a pathway involving activation of protein kinase $\mathrm{C} \varepsilon(\mathrm{PKC} \varepsilon)$, because $\mathrm{Bv} 8$ caused translocation of $\mathrm{PKC} \varepsilon$ to the neuronal membrane and because PKC antagonists reduced both the enhancement of current carried by TRPV1 and behavioral hyperalgesia in rodents. The neuronal population expressing PKRs consisted partly of small peptidergic neurons and partly of neurons expressing the N52 marker for myelinated fibers. Using single-cell reverse transcriptase-PCR, we found that mRNA for PKR1 was mainly expressed in small DRG neurons. Exposure to GDNF (glial cell line-derived neurotrophic factor) induced de novo expression of functional receptors for Bv8 in a nonpeptidergic population of neurons. These results show that prokineticin receptors are expressed in nociceptors and cause heat hyperalgesia by sensitizing TRPV1 through activation of PKC $\varepsilon$. The results suggest a role for prokineticins in physiological inflammation and hyperalgesia.
\end{abstract}

Key words: pain; protein kinase; sensitization; hyperalgesia; substance P; sensory neurons; capsaicin; heat; nociception; nociceptor; TRPV

\section{Introduction}

A small protein isolated from the skin of the frog Bombina variegata causes contraction of guinea pig ileum at nanomolar concentrations (Schweitz et al., 1990; Mollay et al., 1999; Li et al., 2001) and potently induces hyperalgesia in rodents (Mollay et al., 1999; Negri et al., 2002). This peptide was named Bv8 to indicate its origin (Bombina variegata) and its molecular weight $(8 \mathrm{kDa})$. Bv8 belongs to a novel family of secreted proteins, the orthologs of which have been conserved throughout evolution from invertebrates to humans. Two mammalian orthologs of Bv8, murine Bv8 (mBv8) and human Bv8 (hBv8) have been identified (Wechselberger et al., 1999). Li et al. (2001) identified sequences encoding two human proteins similar to Bv8, named prokineticin 1 (PK1; a Bv8-like protein) and prokineticin 2 (PK2; or hBv8). The $\mathrm{Bv} 8 / \mathrm{PK}$ family is composed of small proteins of around $80-90$

Received Sept. 13, 2005; revised March 13, 2006; accepted March 22, 2006.

This work was supported by a Wellcome Trust (United Kingdom) grant to P.A.M., by Italian Ministero Istruzione Universitá e Ricerca Grant Co Finanziamento-PRIN 2004-057339 (L.N., P.M., V.V.), and by Fondazione Cassa di Risparmio di Modena and Fondazione Cassa di Risparmio di Carpi (V.V.). We thank Giorgia Pavesi for her participation in some experiments and for help with data analysis and Ewan St. J. Smith for helpful comments.

Correspondence should be addressed to Peter McNaughton, Department of Pharmacology, Cambridge University, Tennis Court Road, Cambridge CB2 1PD, UK. E-mail: pam42@cam.ac.uk.

DOI:10.1523/JNEUROSCI.3870-05.2006

Copyright $\odot 2006$ Society for Neuroscience $\quad$ 0270-6474/06/265109-08\$15.00/0 amino acids that share a high identity in their amino acid sequence: hPK1 has an overall identity with hPK 2 of $76 \%$ and with amphibian Bv8 of $43 \%$.

PK1 and PK2 are ligands for two G-protein-coupled receptors called prokineticin receptor 1 (PKR1) and PKR2 (Lin et al., 2002; Masuda et al., 2002; Soga et al., 2002). PKR1 and PKR2 have an overall identity in their amino acid sequences of $85 \%$, with most differences at the N-terminal (Lin et al., 2002).

An involvement of the PK/PKR system in nociception is suggested by the observation that systemic injection of Bv8 into rats induces a biphasic hyperalgesia to tactile and thermal stimuli (Negri et al., 2002). The initial phase of hyperalgesia is caused by a local action on nociceptors, because a few femtomoles of Bv8 injected intraplantarly causes a strong and localized hyperalgesia with a similar time course to that of the initial phase of hyperalgesia seen with systemic injection. The secondary phase of hyperalgesia is not seen with local intraplantar injection and is therefore probably attributable to a central action of Bv8.

More recent evidence confirms that activation of the PK/PKR system plays an important role in physiological hyperalgesia. PK2 (i.e., mammalian Bv8) is highly expressed in peripheral blood cells, notably in monocytes, neutrophils, and dendritic cells, and PK2 is released by neutrophils at sites of inflammation, where it can stimulate migration of monocytes (LeCouter et al., 2004). 
Granulocytes also express high levels of PK2 and release it when activated (P. Melchiorri, R. Lattanzi, and L. Negri, personal communication). Martucci et al. (2006) found that Bv8 can activate macrophages, inducing the release of the proinflammatory cytokines interleukin 1 (IL-1) and IL-12. Evidence for a direct involvement of PKR1 in inflammatory hyperalgesia comes from a study of PKR ${ }^{-/-}$mice (L. Negri, R. Lattanzi, E. Giannini, M. Colucci, F. Margheriti, P. Melchiorri, V. Vellani, H. Tian, M. De Felice, and F. Porecca, unpublished data). In this study, the heat and capsaicin sensitivity of PKR $1^{-/-}$mice was found to be significantly lower than wild type (WT), implying that the presence of PKR1 (and presumably its activation by endogenous PK1 or PK2) exerts a tonic activating effect on transient receptor potential vanilloid 1 (TRPV1). Inflammatory heat hyperalgesia caused by mustard oil or CFA was also significantly lower in PKR $1^{-1-}$ mice, suggesting that these nonspecific proinflammatory stimuli act in part via release of $\mathrm{PK}$ and activation of PKR1 receptors.

In the present study, we explored the cellular basis of hyperalgesia caused by PKR activation by investigating the action of Bv8 on isolated nociceptive neurons.

\section{Materials and Methods}

Dorsal root ganglion primary cultures. Neonatal Sprague Dawley rats (3-7 $\mathrm{d}$ of age) were used for the majority of experiments, with adult mice (TO strain) used for some studies as identified below. Rodents were killed by cervical dislocation and decapitation. Dorsal root ganglia (DRGs) were collected, incubated for $1 \mathrm{~h}$ at $37^{\circ} \mathrm{C}$ with $0.125 \%$ collagenase (Worthington, Freehold, NJ), and mechanically dissociated, plated onto coverslips or Petri dishes pretreated with $10 \mu \mathrm{g} / \mathrm{ml}$ poly-L-lysine (Sigma, St. Louis, $\mathrm{MO}$ ) and $20 \mu \mathrm{g} / \mathrm{ml} \mathrm{laminin} \mathrm{(Sigma),} \mathrm{and} \mathrm{cultured} \mathrm{in} \mathrm{DMEM} \mathrm{containing}$ $1 \%$ penicillin/streptomycin, $10 \%$ fetal bovine serum, $1 \%$ L-glutamine (Invitrogen, San Diego, CA), $1.5 \mu \mathrm{g} / \mathrm{ml}$ cytosine $1-\beta$-D-arabinofuranoside (Sigma), and $100 \mathrm{ng} / \mathrm{ml}$ NGF (2.5S NGF; Invitrogen), as described previously (Vellani et al., 2001).

Intracellular calcium imaging. Isolated DRG neurons, plated onto coverslips and cultured 1-3 din vitro, were loaded with the calcium-sensitive fluorescent indicator Fluo-4 AM (10 $\mu$ M; Invitrogen), and calcium imaging was performed as described previously (Vellani et al., 2004). In brief, coverslips were mounted onto an inverted confocal microscope (MicroRadiance; Bio-Rad, Hemel Hempstead, UK) in HBSS (140 mM $\mathrm{NaCl}, 1.8 \mathrm{~mm} \mathrm{CaCl}_{2}, 1 \mathrm{~mm} \mathrm{MgCl}_{2}, 4 \mathrm{~mm} \mathrm{KCl}, 10 \mathrm{~mm}$ HEPES, $4 \mathrm{~mm}$ glucose, $\mathrm{pH}$ 7.4) and were observed using a high numerical aperture $20 \times$ objective. Fluo-4 AM was excited at $488 \mathrm{~nm}$, and images of the fluorescence, $F$, were captured every $4 \mathrm{~s}$ using the Time Course software package (Bio-Rad). Neurons were distinguished from non-neuronal cells at the start of the experiment by applying $25 \mathrm{~mm} \mathrm{KCl}$, which induces a rapid increase of $\left[\mathrm{Ca}^{2+}\right]_{\mathrm{i}}$ in neurons. At the end of the experiment, the maximal fluorescence $\left(F_{\max }\right)$ was obtained by application of the calcium ionophore ionomycin (10 $\mu \mathrm{M}$; Calbiochem, La Jolla, CA) in the presence of $\mathrm{Ca}^{2+}(30 \mathrm{~mm})$ and $\mathrm{K}^{+}(125 \mathrm{~mm})$. Data are expressed as $F / F_{\max }$. All experiments were performed at room temperature (RT) $\left(20-22^{\circ} \mathrm{C}\right)$.

Electrophysiology. All recordings were made from the somata of smalldiameter $(15-25 \mu \mathrm{m})$ DRG neurons from neonatal rats with the wholecell patch-clamp technique at a holding potential of $-70 \mathrm{mV}$ using an Axopatch amplifier and pClamp software (Molecular Devices, Palo Alto, CA) as described previously (Vellani et al., 2001). Only one recording was performed on each culture dish to ensure that data were not obtained from cells that had been inadvertently exposed to other test treatments. All experiments were performed at room temperature $\left(20-22^{\circ} \mathrm{C}\right)$.

Immunocytochemistry. For protein kinase $\mathrm{C} \varepsilon(\mathrm{PKC} \varepsilon)$ visualization, rat DRG neurons cultured for 1-3 d in vitro were treated with Bv8 (100 nM for $30 \mathrm{~s}$ unless otherwise specified) and rapidly fixed for $10 \mathrm{~min}$ at room temperature with paraformaldehyde ( $4 \%$ formaldehyde and $4 \%$ sucrose, dissolved in PBS/distilled water 2:1). Fixed cells were washed three times in PBS (with $0.1 \%$ fish skin gelatin to block nonspecific sites), permeabilized for $30 \mathrm{~min}$ at room temperature with Triton X-100 (0.2\% in PBS), and incubated overnight at $4^{\circ} \mathrm{C}$ with polyclonal anti-PKC $\varepsilon$ antibody
(Schaap et al., 1989) diluted 1:1000 in PBS-T/gelatin (PBS with $0.05 \%$ Triton X-100). Coverslips were then incubated for $1 \mathrm{~h}$ at room temperature with goat anti-rabbit IgG conjugated to the fluorophore Alexa Fluor 488 (1:200; Invitrogen), washed three times in PBS/gelatin, and visualized in the confocal microscope.

To characterize subpopulations of Bv8-activated neurons, double immunostaining on $3 \mathrm{~d}$ DRG cultures was performed. Coverslips processed for PKC $\varepsilon$ immunoreactivity as above were incubated overnight at $4^{\circ} \mathrm{C}$ with the following polyclonal antibodies: anti-substance P (SP), anticalcitonin gene-related peptide (CGRP), or anti-parvalbumin (1:100; Invitrogen) or anti-N52 (1:100; Santa Cruz Biotechnology, Santa Cruz, CA). After washing, coverslips were exposed for $1 \mathrm{~h}$ at room temperature to donkey anti-goat conjugated to the fluorophore Alexa Fluor 594 (1: 200; Invitrogen), washed three times in PBS/gelatin, and visualized in the confocal microscope. Double staining for IB4, on coverslips previously processed for $\mathrm{PKC} \varepsilon$, was assessed by incubating the cells for $1 \mathrm{~h}$ at room temperature with IB4 bound to Alexa Fluor 594 (1:100; Invitrogen) followed by washing (three times). Coverslips were stored at $4^{\circ} \mathrm{C}$ in sodium azide $(0.5 \%$ in PBS) for additional analysis.

Laser microdissection. Adult rat DRG cells, isolated following the protocol described above, were centrifuged ( $5 \mathrm{~min} ; 500 \mathrm{rpm}$ ) onto a coverslip coated with a polyethylene naphthalate membrane (PALM MembraneSlides; P.A.L.M., Munich, Germany), previously activated by UV light for $30 \mathrm{~min}$. Cells were immediately fixed in 70\% ethanol for $10 \mathrm{~min}$ at $-20^{\circ} \mathrm{C}$ and stored at $-80^{\circ} \mathrm{C}$. For single-cell capture, coverslips were mounted onto an inverted microscope and visualized with a $40 \times$ objective. Neurons were flattened after centrifugation, and diameters did not relate to neuronal sizes in culture (see Fig. $1 D$ ). Cells were therefore classified into three classes containing approximately equal numbers (see Fig. 5), and coordinates were registered by an automated system (PALM Microlaser Technologies). The cap of a microvial containing $40 \mu$ lof lysis buffer (RNeasy Min Elute Cleanup Kit; Qiagen, Hilden, Germany) was inserted over the coverslip, and 70-200 identified cells from a single class were ejected by the laser microdissection and pressure catapulting technique. Collected samples were stored at $-80^{\circ} \mathrm{C}$ and analyzed by one-step real-time reverse transcription (RT)-PCR.

One step real-time RT-PCR. Total RNA extraction from samples collected by laser microdissection were performed using RNeasy columns (Qiagen). Approximately $0.5 \mathrm{ng}$ of RNA was reverse-transcribed and amplified following the quantitative one-step real-time RT-PCR technique (QuantiTect SYBR Green RT-PCR; Qiagen), with both RT and PCR in the same tube. Specific sense and antisense primers were synthesized to amplify the cDNA corresponding to rat PKR1 and PKR2, according to the following sequences: for PKR1, 5'-CGCACCGTCTCCCTCTAC-3' and 5'-GTTTGACACTTCATCCGCG-3'; for PKR2, 5'

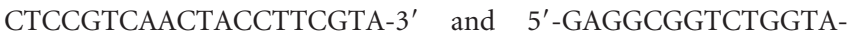
ATTCA-3'. RT-PCRs were performed using an iCycler thermal cycler (Bio-Rad) with the following protocol: $30 \mathrm{~min}$ at $50^{\circ} \mathrm{C}$ for the RT; $15 \mathrm{~min}$ at $95^{\circ} \mathrm{C}$ for the inactivation of the reverse transcriptase; 50 PCR amplification cycles; $15 \mathrm{~s}$ at $94^{\circ} \mathrm{C}$ for the denaturation; $30 \mathrm{~s}$ at $52^{\circ} \mathrm{C}$ for the annealing; $30 \mathrm{~s}$ at $72^{\circ} \mathrm{C}$ for the extension of the amplification products. The iCycler registered in real time the fluorescence produced by the fluorophore SYBR Green I (Invitrogen) after its intercalation into PCR products. The threshold cycle is defined as the point at which the fluorescence rises appreciably above background. Because there is a linear relationship between the log of the starting amount of template and the corresponding threshold cycle during real-time PCR, it was possible to construct a standard curve that could be used to interpolate the starting amount of template based on its threshold cycle. The comparison of the threshold against the gene of interest (normalized for the threshold cycle of the housekeeping gene $\beta$-actin), assessed on tissues or different samples, allowed the level of relative expression to be determined.

Measurements of nociception in vivo. Responses to noxious heat were measured in adult male Sprague Dawley rats (220-250 g) and to capsaicin in adult male Swiss mice (30-35 g) after intraplantar injection of Bv8 or saline injected into the rat $(40 \mu \mathrm{l})$ and mouse $(20 \mu \mathrm{l})$ paw with a microsyringe fitted with a 30 gauge needle. All experiments were performed according to protocols approved by the Animal Care and Use Committee of the Italian Ministry of Health according to European 

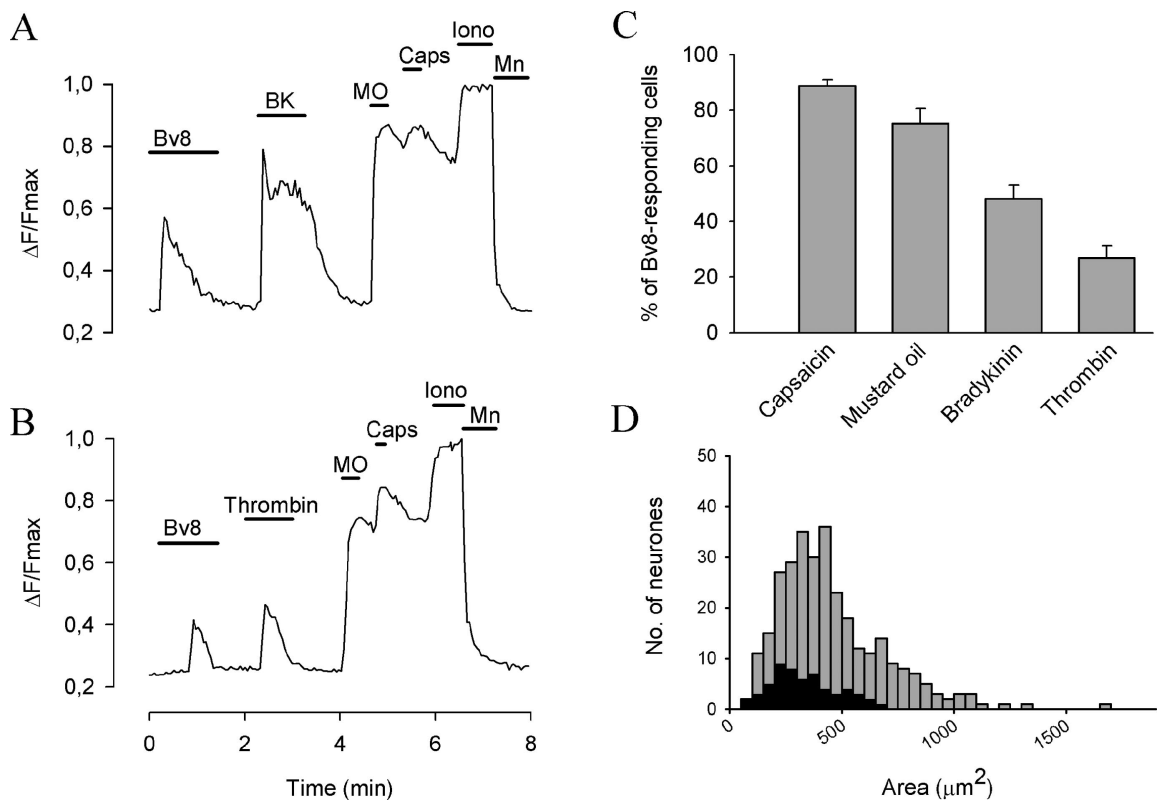

Figure 1. Increases in intracellular calcium in response to application of Bv8. $A, B$, Time course of change in calcium-dependent fluorescence during application of Bv8 (100 nM), bradykinin (1 $\mu \mathrm{M})$, mustard oil (1 $\mu \mathrm{M})$, capsaicin $(500 \mathrm{nM})$, and thrombin (100 nM) as shown. $\boldsymbol{C}$, Percentage of Bv8-responsive neurons also responding to other agonists. $\boldsymbol{D}$, Distribution of diameters of neurons responding to Bv8 (black; mean area, $355 \mu \mathrm{m}^{2}$ ) compared with the total neuronal population (gray; mean area, $453 \mu \mathrm{m}^{2}$ ). №., Number.

Community directives. Animals were used only once throughout the experiments.

Capsaicin-related pain behavior was evaluated in mice. Capsaicin $(0.01 \mathrm{nmol} / 10 \mu \mathrm{l})$ was injected into the plantar skin (intraplantar injection) of the hindpaw of mice $30,60,90,150,240$, or 360 min after the injection of $0.2 \mathrm{pmol}$ of Bv8 or saline. Animals were placed individually in chambers (transparent glass cylinders of $20 \mathrm{~cm}$ in diameter) and were adapted for $30 \mathrm{~min}$ before capsaicin or vehicle injection. After challenge, mice were observed individually for $20 \mathrm{~min}$. The total amount of time spent licking the injected paw was timed with a chronometer.

The withdrawal time to localized thermal stimuli was measured on the paws of freely moving rats with the Hargreaves' plantar test (Ugo Basile, Comerio, Italy). Rats were trained in paw-withdrawal tests during the week preceding the experiment at $30 \mathrm{~min}$ intervals for $3 \mathrm{~h}$ daily. This adaptation protocol reduced variability in threshold measurements, giving a more stable baseline and making drug-induced changes easier to detect. On the day of the experiment, withdrawal time was measured for $2 \mathrm{~h}$ at $30 \mathrm{~min}$ intervals before drug injection. The mean of the last three of these threshold measurements was taken as baseline nociceptive threshold $\left(\mathrm{NT}_{\mathrm{B}}\right)$. Nociceptive threshold was then determined three times at each of 15, 30, 60, 90, 120, 150, and 180 min after saline or Bv8 solution injection. The mean of the three readings at each time point was defined as the nociceptive threshold at that time in the presence of the test solution $\left(\mathrm{NT}_{\mathrm{ts}}\right)$. The effect of the test drug was calculated as the percentage change in nociceptive threshold from baseline threshold $(\% \Delta \mathrm{NT})$ according to the following equation: $\% \Delta \mathrm{NT}=100 \times\left(\mathrm{NT}_{\mathrm{ts}}-\mathrm{NT}_{\mathrm{B}}\right) / \mathrm{NT}_{\mathrm{B}}$.

Materials. Bv8 was isolated from the skin secretions of the frog Bombina variegata and purified to $98 \%$ by HPLC, according to the method of Mollay et al. (1999). Glial cell line-derived neurotrophic factor (GDNF) was obtained from Preprotech EC (London, UK), NGF was obtained from Promega (Madison, WI), and other chemicals were obtained from Sigma unless otherwise stated.

Statistics. All values in the text or in graphs are given as mean \pm SEM. Statistical analyses were performed using one-way ANOVA followed by Tukey's multiple comparison posttest or Student' test, as appropriate.

\section{Results \\ Bv8 mobilizes intracellular calcium in nociceptive neurons}

Bv8 at nanomolar concentrations elicited robust increases in intracellular calcium in a subpopulation of DRG neurons from both mice and rats (Fig. 1A,B) (Negri et al., 2002). A calcium signal induced by 100 $\mathrm{nm}$ Bv8 was seen in $16 \pm 1.4 \%$ of neuronal cells from neonatal rats $(n=49$ coverslips from 16 different cultures). The calcium signal was typically elicited with a substantial delay: the rise in calcium started with a latency of $19.7 \pm 1.3 \mathrm{~s}$ (range, 3-55 s) after exposure to Bv8, but once the calcium increase had started, it grew steeply and quickly reached a peak (within $32.6 \pm 2.6 \mathrm{~s}$ from Bv8 application; range, 9-81 s). The average peak amplitude was $0.31 \pm 0.022$ $\Delta F / F_{\max }$ (range, $0.1-0.92$ ), and after the peak, the calcium response to Bv8 recovered to a value of $50 \%$ of the peak amplitude within $66.0 \pm 3.9 \mathrm{~s}$ after Bv8 application (range, 24-140 s). Calcium signals in response to Bv8 were present in a significantly lower number of neurons in adult mice $(7 \pm 1.1 \% ; n=12$ coverslips from six different cultures).

Neurons responding to Bv8 were predominantly of small and medium diameter (Fig. $1 D$ ). In neurons from neonatal rats, the mean area was $355 \pm 22 \mu \mathrm{m}^{2}$, compared with an average of $453 \pm 14 \mu \mathrm{m}^{2}$ for the whole neuronal population. In adult mice, the Bv8 responding population had an average area of $474 \pm 13 \mu \mathrm{m}^{2}$, and the whole neuronal population had a average area of $365 \pm 6 \mu \mathrm{m}^{2}$ (nine coverslips in three cultures), showing that in mice, there is a greater tendency than in rats for functional PKRs to be found in the large-diameter neuronal population.

The colocalization of PKRs with ion channels and receptors characteristic of nociceptive neurons was investigated by applying appropriate agonists (Fig. 1). Of the neurons responding to Bv8, $88.9 \pm 2.1 \%$ (19 coverslips in seven experiments) also responded to capsaicin (Fig. 1C), showing a high degree of colocalization of functional PKRs with the heat and capsaicin-activated ion channel TRPV1 (Caterina et al., 1997). Responses to Bv8 were colocalized with responses to mustard oil, an activator of the channel TRPA1, which like TRPV1 is involved in nociception (Tominaga and Caterina, 2004). We also found substantial colocalization of responses to Bv8 with G-protein-coupled receptors for bradykinin (48.3 $\pm 4.0 \%$; six coverslips in six experiments) and thrombin, which activates protease-activated receptors by cleavage to expose a tethered ligand $(26.8 \pm 4.4 \%$; seven coverslips in six experiments). The calcium responses generated by Bv8 had a different time course from those generated by bradykinin and thrombin: the delay to initiation of the response to Bv8 of $19.7 \mathrm{~s}$ (see above) was significantly longer than for bradykinin $(9.6 \pm 0.7 \mathrm{~s} ; 17$ coverslips in six experiments; $p<0.001)$ but shorter than for thrombin $(32.6 \pm 1.6 \mathrm{~s} ; p<0.001)$.

Very few non-neuronal cells responded to Bv8 with a calcium increase ( $\sim 1$ in 5000 in adult mouse DRG cultures; none in neonatal rat cultures). Non-neuronal cells were identified from their flattened morphology and small size and by the absence of an 


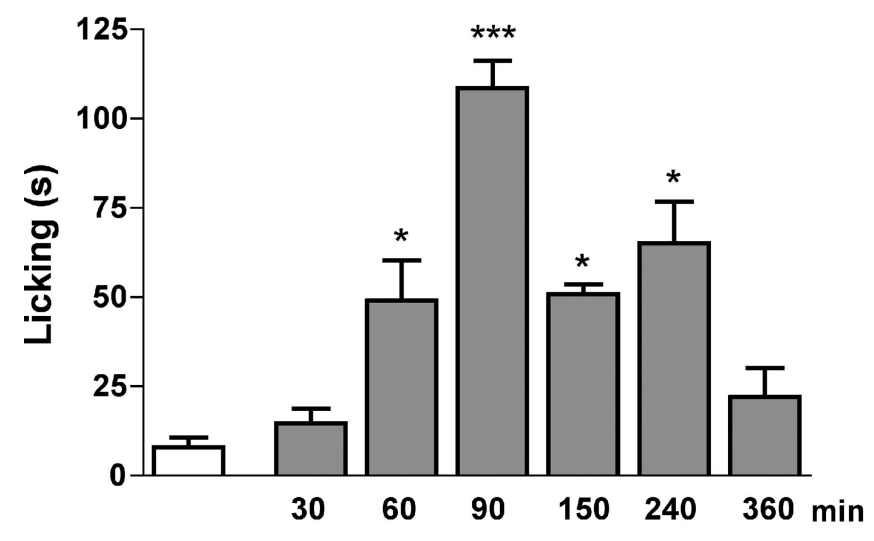

Bv8 50 fmol, i.pl.

Figure 2. Bv8 potentiates the effect of capsaicin in vivo. Licking evoked by intraplantar injection (i.pl.) of $0.01 \mathrm{nmol}$ capsaicin $30 \mathrm{~min}$ after injection of saline (white column) or at the stated times after intraplantar injection of $50 \mathrm{fmol}$ of Bv8 in the same volume of saline. The effect is expressed as total time spent licking the injected paw. Results are mean \pm SEM from four to five animals. ${ }^{*} p<0.05,{ }^{* * *} p<0.001$ versus saline.

increase in $\left[\mathrm{Ca}^{2+}\right]_{\mathrm{i}}$ after application of $25 \mathrm{mM}\left[\mathrm{K}^{+}\right]$, which activates calcium channels in neurons but not in non-neuronal cells. Non-neuronal cells were capable of generating robust calcium signals, however, because the majority responded vigorously to endothelins (data not shown), showing that the absence of a calcium response to Bv8 was not a result of a defect in calcium release pathways. The almost complete absence of a calcium signal in response to Bv8 in non-neuronal cells argues against the possibility that PKRs are located on non-neuronal cells, which then activate neurons by release of a secondary factor.

Bv8 sensitizes the heat and capsaicin-gated channel, TRPV1

Activation of PKRs with Bv8 causes heat hyperalgesia in vivo (Negri et al., 2002). Several mechanisms are thought to be responsible for detecting heat stimuli (Caterina et al., 1999; Caterina et al., 2000; Davis et al., 2000; Woodbury et al., 2004), but of these only TRPV1 is activated by capsaicin, as shown by the complete ablation of response to capsaicin in animals in which the gene for TRPV1 has been deleted (Caterina et al., 2000; Davis et al., 2000). We therefore investigated whether activation of PKRs sensitized the response to capsaicin in vivo to determine whether sensitization of TRPV1 underlies the heat hyperalgesia caused by Bv8. Intraplantar administration of capsaicin resulted in a doserelated intermittent paw licking that lasted 10-15 min. A just suprathreshold dose of $0.01 \mathrm{nmol}$ capsaicin induced licking for a total of $8 \pm 2.7 \mathrm{~s}$, but after injection of Bv8, a significant enhancement of licking of the paw was observed (Fig. 2). The effect had disappeared by $6 \mathrm{~h}$ after Bv8 administration. Bv8 administration by itself caused no observable behavioral effects. These results show that Bv8 does indeed sensitize TRPV1, and that part or all of the thermal sensitization caused by Bv8 is attributable to potentiation of TRPV1, but they do not rule out the possibility that other heat-detecting ion channels may also be sensitized.

In subsequent experiments on isolated nociceptive neurons, we used capsaicin as a specific agonist to activate TRPV1. Application of Bv8 did not elicit detectable inward current in DRG neurons, consistent with behavioral experiments showing that injection of Bv8 per se did not produce pain in the intact animal (Negri et al., 2002). In a subpopulation of neurons, however, exposure to Bv8 produced a dramatic increase in the capsaicinactivated current (Fig. $3 A, B$ ). In other neurons, Bv8 had no sen-
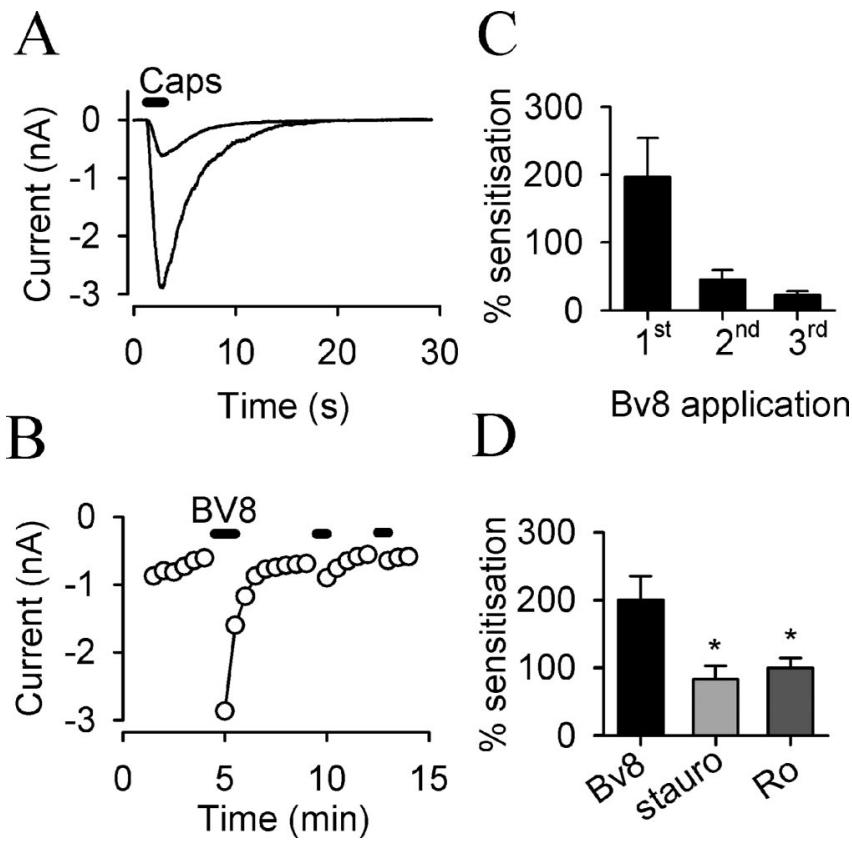

Figure 3. Bv8 sensitizes the capsaicin-gated inward current. $\boldsymbol{A}$, Inward current activated by a 1 s pulse of capsaicin ( $500 \mathrm{~nm}$ ) is enhanced by Bv8 ( $100 \mathrm{~nm} ; 30$ s application). $\boldsymbol{B}$, Time course of sensitization of capsaicin-activated inward current by Bv8. Some sensitization was observed even after repeated application. C, Sensitization of inward current in Bv8-responding cells with repeated exposure to Bv8; mean of eight experiments similar to that shown in $\boldsymbol{B}$. D, Sensitization by Bv8 is partially blocked by the kinase inhibitor staurosporine $(1 \mu \mathrm{M})$ and the PKC-specific inhibitor R0 31-8220 (100 nm). * $p<0.05$.

sitizing effect on the response to capsaicin, showing that not all TRPV1-expressing neurons express PKRs. The sensitizing effect of Bv8 was observed to be markedly but not totally attenuated after repeated exposure (Fig. $3 C$ ).

\section{PKC inhibitors reduce sensitization of TRPV1 by Bv8}

The Bv8-induced sensitization of the response to capsaicin in isolated DRG neurons was partially inhibited by previous application of staurosporine, a relatively nonspecific kinase inhibitor, and by RO-318221 (Calbiochem, Nottingham, UK), a specific inhibitor of PKC (Fig. 3D). These results show that PKC is an important participant in sensitization caused by Bv8 but suggest that other cellular signaling pathways are also involved.

The sensitization caused by Bv8 was also found to be reduced by $\mathrm{PKC}$ inhibitors in in vivo experiments on rats (Fig. 4). When 0.2 pmol Bv8 was injected into one rat hindpaw, a dose that left the nociceptive threshold of the contralateral paw unchanged, the local nociceptive threshold to radiant heat was reduced by 45 $50 \%$. This local response reached a maximum at $60 \mathrm{~min}$ and recovered after $2-3 \mathrm{~h}$. The decrease of heat threshold was abolished by intraplantar administration of the specific PKC inhibitors RO-318221 and bisindolylmaleimide I (Fig. 4).

\section{Bv8 causes translocation of PKC $\varepsilon$ in a subpopulation of nociceptive neurons}

Bradykinin causes activation of $\mathrm{PKC} \varepsilon$, which can be detected by observing the translocation of PKC $\varepsilon$ from the cytoplasm to the neuronal cell membrane (Cesare et al., 1999; Vellani et al., 2004). Activated PKC $\varepsilon$ sensitizes TRPV1 by phosphorylating two serine residues (Numazaki et al., 2002). We found that Bv8 also caused translocation of PKC- $\varepsilon$ to the neuronal cell membrane (Fig. $5 A, B)$. After exposure to $100 \mathrm{nM} \mathrm{Bv8}$, the proportion of rat 


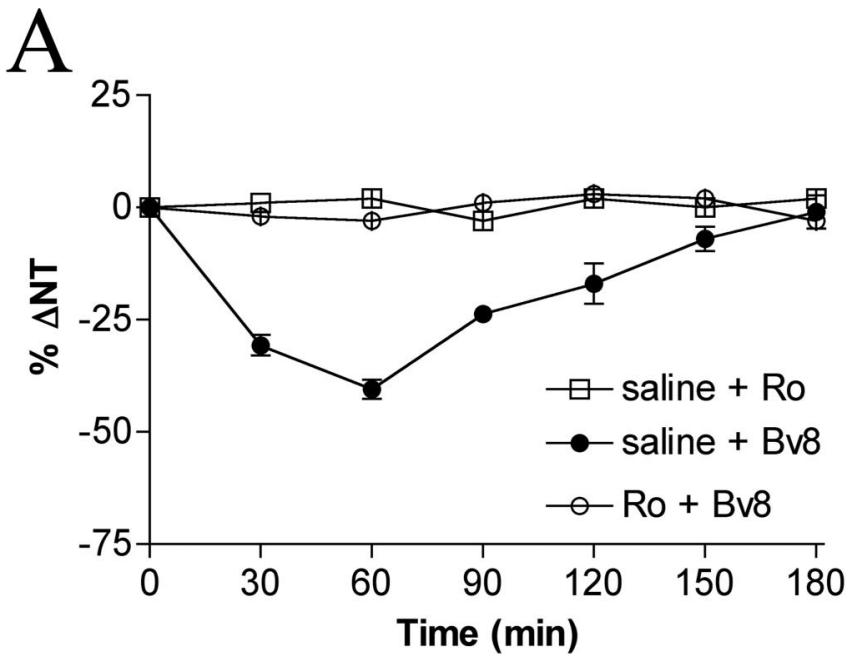

B

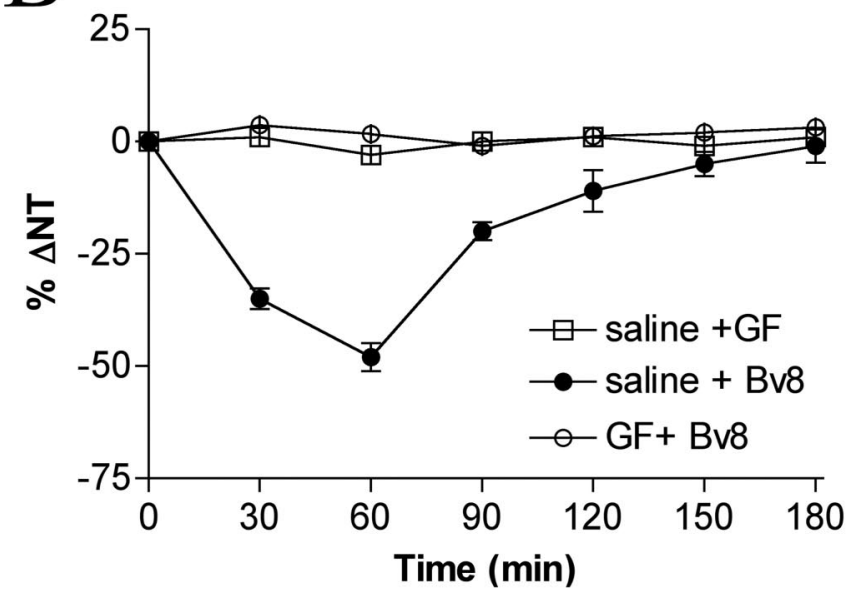

Figure 4. PKC inhibitors abolish heat hyperalgesia induced by Bv8. Reduction of heat threshold measured using Hargreaves plantar test in rats (see Materials and Methods). A, Bv8 alone caused substantial heat hyperalgesia (paw-removal time decreased from $12 \pm 1.3$ to $7.2 \pm 1 \mathrm{~s}$ ) and intrapaw injection of R0 31-8220 (Ro; $10 \mu \mathrm{g}$; Calbiochem) 5 min before the injection of 0.2 pmol Bv8 completely abolished the Bv8-induced thermal hyperalgesia. The R0 31-8220 dose chosen, when injected by itself, leaves the baseline nociceptive threshold (NT) unchanged. $\boldsymbol{B}$, Similar experiments using the specific PKC inhibitor bisindolylmaleimide I [GF 109203 (GF), 2 $\mu \mathrm{g}$; Calbiochem].

neurons exhibiting translocation peaked at $18.8 \pm 0.95 \%$ (35 coverslips) (Fig. $5 D, E$ ), a proportion similar to that in which calcium signals are elicited by Bv8 (see above). With longer exposures to $\mathrm{Bv} 8, \mathrm{PKC} \varepsilon$ was observed to be internalized in vesicles in a substantial number of cells (Fig. $5 C$ ), as seen after longer exposures to bradykinin (Cesare et al., 1999). The percentage of cells in the mouse showing translocation of PKC $\varepsilon$ in response to Bv8 was smaller than in the rat $(7.8 \pm 0.5 \%$ after $3 \mathrm{~d}$ in culture; 13 coverslips from six cultures), consistent with the smaller proportion of mouse neurons in which calcium signals were observed (see above).

The relationship between Bv8 concentration and the proportion of cells in which PKC $\varepsilon$ is translocated after a $30 \mathrm{~s}$ exposure was well fitted at lower concentrations by a first-order equation with half-maximal activation at $0.65 \mathrm{~nm}$ Bv8 (Fig. 5D). The proportion of cells showing PKC $\varepsilon$ translocation peaked at $100 \mathrm{~nm}$ Bv8, and at higher concentrations, the proportion of cells showing translocation decreased. The reason for this decrease is not
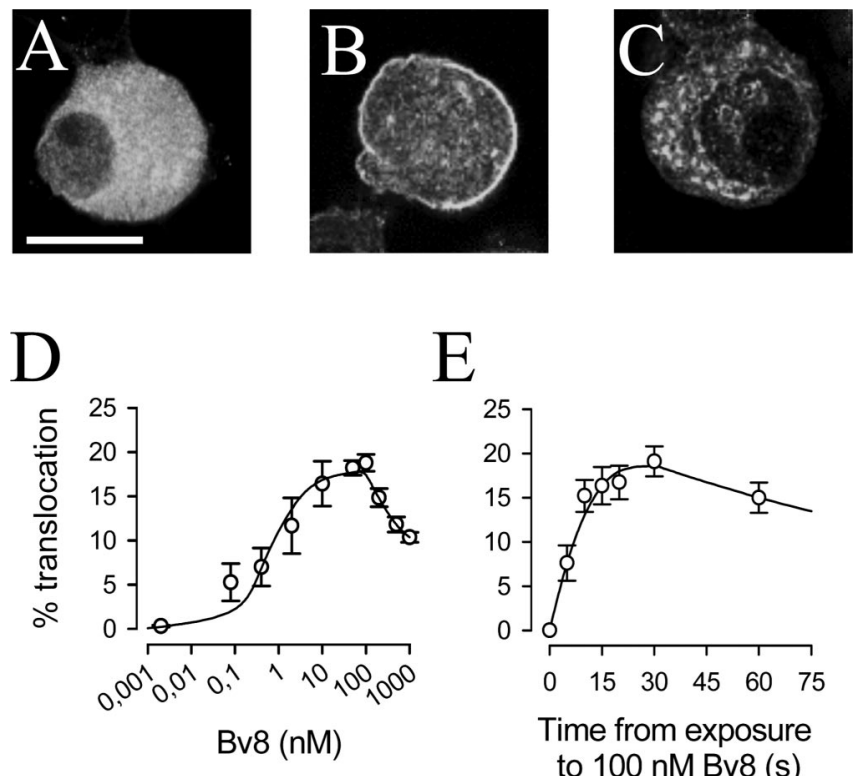

E

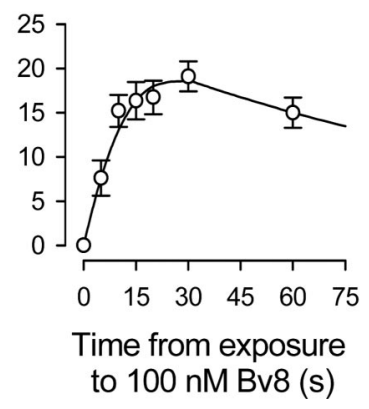

Figure 5. Bv8 causes translocation of PKC $\varepsilon$ to the neuronal membrane. $\boldsymbol{A}-\boldsymbol{C}$, Confocal images of neurons labeled with PKC $-\varepsilon$. $\boldsymbol{A}$, Control. No neurons showing PKC $\varepsilon$ translocation were seen in control preparations, and none showed PKC $\varepsilon$ internalization. Scale bar, $\boldsymbol{A}-\boldsymbol{C}, 20 \mu \mathrm{m}$. $\boldsymbol{B}$, Neuron showing PKC $\varepsilon$ translocation to the surface membrane 30 s after exposure to $100 \mathrm{~nm} \mathrm{Bv8.}$ C, Neuron showing PKC $\varepsilon$ internalization after exposure to Bv8. D, Percentage of neurons showing PKC $\varepsilon$ translocation as a function of Bv8 concentration ( 30 s exposure). Curve between 0 and $100 \mathrm{~nm}$ [Bv8] shows a first-order equation fitted to the data with half-activation at $0.65 \mathrm{~nm}$ [Bv8]. $\boldsymbol{E}$, Effect of time of exposure to $100 \mathrm{~nm}$ Bv8.

clear but may involve a reduction in the effective concentration of Bv8 through complex formation.

The time course of translocation of PKC $\varepsilon$ was also investigated (Fig. $5 E$ ). The proportion of cells showing PKC $\varepsilon$ translocation increased rapidly, reaching a peak at $30 \mathrm{~s}$ and declining thereafter as PKC $\varepsilon$ is internalized. The maximum translocation was consistently observed with $100 \mathrm{nM}$ Bv8 and a 30 s exposure, and this concentration and time of exposure was therefore adopted for subsequent immunocytochemistry experiments.

\section{Characteristics of neurons expressing functional prokineticin receptors}

Nociceptive neurons can be divided into three major classes: those subtending myelinated $\mathrm{A} \delta$ fibers and expressing markers for myelination such as neurofilament $\mathrm{H}$ (Lee and Cleveland, 1996), those subtending unmyelinated $C$ fibers and expressing the neuropeptides SP and CGRP, and those subtending C fibers and negative for SP and CGRP but expressing surface glycoproteins binding the lectin IB4 (Snider and McMahon, 1998). We used PKC $\varepsilon$ translocation in response to application of Bv8 as an index of expression of functional prokineticin receptors and costained for IB4, SP, CGRP, and neurofilament $\mathrm{H}$ to examine colocalization of PKRs with other nociceptive neuronal markers (Fig. 6A-E). The large majority of neurons expressing functional PKRs were IB4-negative (Fig. 6G) (IB4-positive cells showing PKC $\varepsilon$ translocation comprised $1.4 \pm 1.1 \%, n=8$, of the total in the neonatal rat and $0.9 \pm 0.5 \%, n=4$, in the adult mouse). This result suggested that functional PKRs would be expressed in the peptidergic subpopulation of DRG neurons. Double-labeling experiments confirmed that CGRP was indeed present in approximately one-third of the neurons expressing functional PKRs (Fig. $6 C, G$ ) and SP in approximately one-fifth (Fig. 6D, G). However, a significant percentage of neurons that are IB4 negative and 

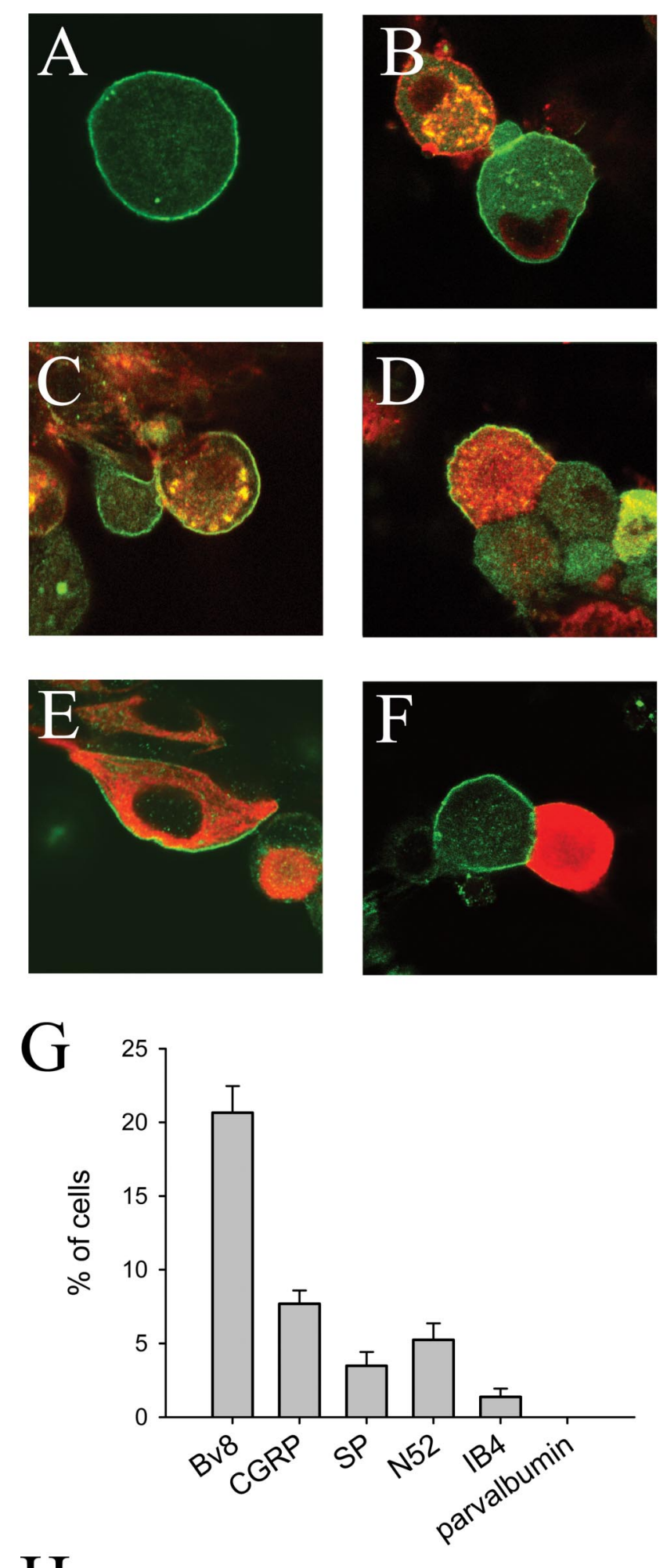

$\mathrm{H}$
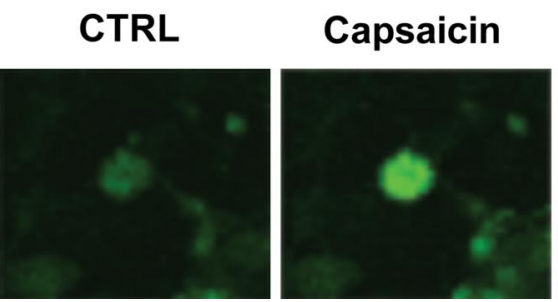

\section{Anti-N52}

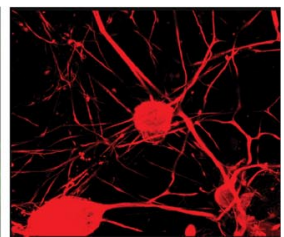

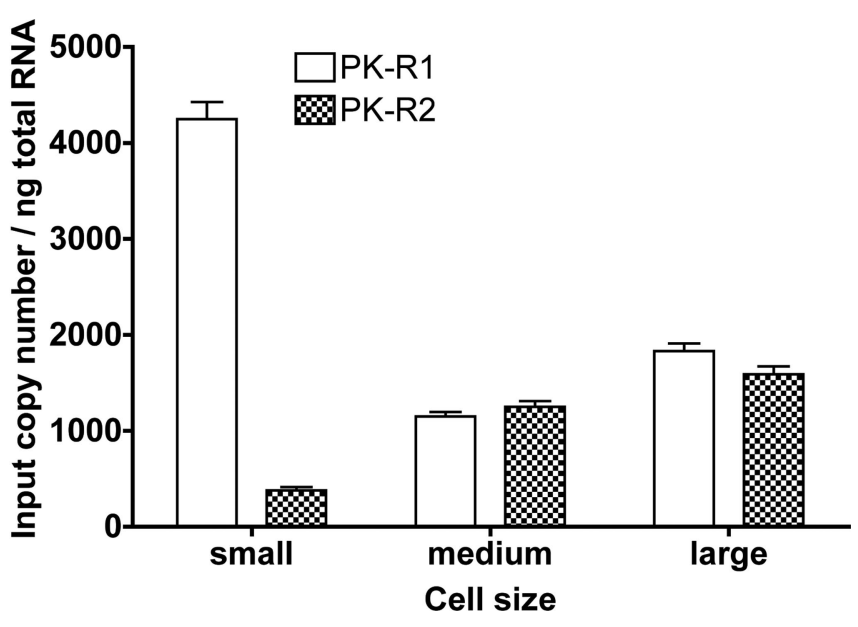

Figure 7. Distribution of PKR1 and PKR2 mRNA in DRG neuronal size classes. Expression of PKR1 (black) and PKR2 (gray) mRNA in adult mouse DRG neurons was determined by single-cell RT-PCR analysis as described in Materials and Methods. Cells were divided into three groups on the basis of neuronal diameter. Data in each group were normalized for total RNA.

nonpeptidergic remained. The N52 monoclonal antibody, which binds with high specificity to neurofilament $\mathrm{H}(\mathrm{NFH})$, is a marker of neurons giving rise to myelinated fibers, and a significant fraction of $\mathrm{NFH}^{+}$neurons showed Bv8-induced translocation of PKC- $\varepsilon$ (Fig. $6 E, G$ ). We examined the proportion of $\mathrm{NFH}^{+}$neurons that expressed functional TRPV1, as assessed by their responses to capsaicin (Fig. $6 \mathrm{H}$ ), and found that $15 \pm 2 \% \mathrm{of} \mathrm{NFH}^{+}$ neonatal rat neurons responded to capsaicin. We also examined parvalbumin (Fig. $6 F$ ), a protein expressed in non-nociceptive sensory neurons innervating muscle spindles (Copray et al., 1994). Parvalbumin and functional PKRs were not coexpressed in any neurons, in support of other evidence (Fig. 1C) that PKRs are expressed mainly in nociceptive DRG neurons.

Functional PKRs are therefore expressed mainly in myelinated and peptidergic unmyelinated sensory neurons. This conclusion agrees with other data (Fig. $1 D$ ) showing that functional PKRs are found in both a small- and a medium-diameter population of DRG neurons.

\section{Expression of mRNA for PKR1 and PKR2}

The expression of mRNA for PKR1 and PKR2 in DRG neurons isolated by the same procedure as used for the functional studies was explored by using single-cell laser capture to separate populations of neurons belonging to three major size classes (Fig. 7). These experiments showed, in agreement with functional data, that PKR1 is expressed predominantly in small DRG neurons, corresponding mainly to neurons subtending unmyelinated axons, but that some expression can be observed in larger neurons.

GDNF upregulates functional responses to Bv8

The results above show that very few neurons expressing functional PKRs belong to the IB4 ${ }^{+}$population, in which the RET

\section{$\leftarrow$}

Figure 6. Colocalization of PKR expression with neuronal markers. $A$, Translocation of PKC $\varepsilon$ after exposure to Bv8 (100 nm; 30 s). $\boldsymbol{B}-\boldsymbol{F}$, Double staining of PKC $\varepsilon$ (green; as in $\boldsymbol{A}$ ) and other neuronal markers, as noted (red). $G$, Percentage of neurons showing translocation of PKC $\varepsilon$ in response to Bv8 (first bar), and proportions of total neurons that both show translocation of Bv8 and are positive for the neuronal markers listed (subsequent bars). $\boldsymbol{H}$, First two panels show a neuron expressing functional TRPV1, as shown by response to capsaicin in a calcium imaging experiment. The same neuron was fixed, and expression of NFH was examined with N52 (last panel). (TRL, Control. 
receptors for GDNF are found. However, we found that expression of functional PKRs is upregulated in the IB $4^{+}$population by exposing neurons to GDNF ( $50 \mathrm{ng} / \mathrm{ml}, 3 \mathrm{~d}$ ). In these experiments, the total proportion of sensory neurons expressing functional PKRs, as assessed by translocation of PKC- $\varepsilon$, was increased significantly by exposure to GDNF (from $17.4 \pm 1$ to $25.8 \pm 1 \%$, an increase of $8.4 \% ; p<0.001$ ). The change was mainly accounted for by an increase in PKR expression in the IB4-positive subpopulation of cells, as the proportion of total neurons that were both $\mathrm{IB}_{4}{ }^{+}$and expressed functional PKRs increased from $1.2 \pm$ $0.32 \%$ in the absence of GDNF to $6.4 \pm 0.6 \%$ when exposed to GDNF (significant difference; $p<0.001$ ). These experiments show that GDNF is able to promote the de novo appearance of receptors for $\mathrm{Bv} 8$ in the $\mathrm{IB}_{4}{ }^{+}$population of DRG neurons, a result that is reminiscent of the ability of GDNF to promote the de novo expression of $\mathrm{B} 1$ receptors for bradykinin in the same neuronal population (Vellani et al., 2004).

\section{Discussion}

The prokineticin/prokineticin receptor system has been discovered only recently, and its biological functions are still mostly unexplored. One clue to a possible function came from experiments in which Bv8, a protein secreted by frog skin and homologous to PK2, was found to produce a profound thermal and mechanical hyperalgesia when injected in small quantities into the paws of rats (Negri et al., 2002). This effect of Bv8 is mediated by an action on nociceptive nerve terminals, rather than being an effect of central origin, because it is localized to the site of injection. Other experiments (see Introduction) show that the prokineticin system plays a physiological role in inflammatory hyperalgesia, because PK2 is released from neutrophils and granulocytes on activation, and because thermal hyperalgesia caused by nonspecific inflammatory stimuli is attenuated by deletion of the gene coding for PKR1 (L. Negri, R. Lattanzi, E Giannini, M. Colucci, F. Margheriti, P. Melchiorri, V. Vellani, H. Tian, M. De Felice, and F. Porecca, unpublished data). However, these results do not provide any evidence for the mechanism of action of Bv8, which could be either direct on nociceptive nerve terminals or an indirect action on surrounding cells, which in turn release a proinflammatory mediator such as bradykinin or a prostaglandin.

In the present study, we investigated the cellular basis of the effect of Bv8 by examining its actions on isolated sensory neurons. We found that Bv8 causes a calcium signal in $\sim 18 \%$ of neurons from neonatal rats and $7 \%$ of neurons from adult mice, and that a calcium signal was detected in very few of the satellite cells that are inevitably present in preparations of cultured DRG neurons. These observations imply that the action of Bv8 is direct on PKRs expressed in neurons, a conclusion supported by the expression of mRNA for both PKR1 and PKR2 in isolated sensory neurons (Fig. 7).

Calcium signals activated by Bv8 in sensory neurons are colocalized with a number of functional markers for nociceptive neurons (Fig. 1). The most striking colocalization is with the heat and capsaicin-gated ion channel, TRPV1, which is coexpressed in 90\% of Bv8-responsive neurons. There was also a high degree of colocalization with responses to mustard oil, an agonist for the ion channel TRPA1 (Bandell et al., 2004; Jordt et al., 2004) and for receptors for bradykinin and thrombin. All of these agonists are associated with pain or hyperalgesia, and their receptors are typically expressed in nociceptive neurons. These observations show that PKRs are located on neurons serving a nociceptive function.

The thermal hyperalgesia observed in vivo after injection of
Bv8 suggests an action on the heat- and capsaicin-gated ion channel, TRPV1. Heat activates several ion channels of the TRP family (Tominaga and Caterina, 2004), leaving open the possibility that the action of Bv8 is via another thermal TRP channel. However, capsaicin specifically activates TRPV1 (Caterina et al., 2000; Davis et al., 2000), and in the present study, we showed that Bv8 potentiates capsaicin-evoked behavioral responses (Fig. 2), confirming that PKR activation potentiates TRPV1 function in vivo. Patch-clamp experiments showed that the ion current activated by capsaicin was enhanced by exposure of neurons to Bv8 (Fig. 3). The sensitization caused by Bv8 both in isolated neurons and in vivo is reduced by $\mathrm{PKC}$ inhibitors, showing that cellular signaling pathways activated by Bv8 involve PKC.

We used translocation of PKC $\varepsilon$ to the neuronal cell membrane as an index of expression of functional receptors for Bv8 (Fig. 5). Translocation was seen in $\sim 18 \%$ of neonatal rat and $8 \%$ of adult mouse neurons, proportions similar to those in which calcium signals are observed. Bv8 was an extremely potent activator of $\mathrm{PKC} \varepsilon$, with $0.65 \mathrm{nM}$ Bv8 half-activating translocation. We next investigated the colocalization of a number of cytochemical markers with the presence of functional receptors for Bv8. The large majority of Bv8-responsive cells were either peptidergic (i.e., positive for CGRP or substance P) or expressed neurofilament $\mathrm{H}$, which is present in cells subtending myelinated fibers (Fig. 6). The presence of receptors for Bv8 in more than one neuronal population is also supported by the observation that calcium signals were observed in both small- and mediumdiameter neurons in response to Bv8 (Fig. 1), and that mRNA for PKR1 was present in both small- and larger-diameter neurons (Fig. 7). TRPV1 is often thought to be expressed mainly in $\mathrm{C}$ fibers, and because TRPV1 and responses to Bv8 are highly colocalized, the expression of functional PKRs in a population of myelinated may therefore appear contradictory. There is, however, clear evidence for expression of TRPV1 in neurons giving rise to myelinated fibers both in previous immunocytochemical studies (Ma, 2002; Amaya et al., 2003; Rashid et al., 2003) and in functional experiments reported here (Fig. $6 \mathrm{H}$ ).

Very few Bv8-responsive cells bound IB4, a marker for the nonpeptidergic population of nociceptive neurons. However, we found that culture with GDNF caused an upregulation in the proportion of IB4 ${ }^{+}$cells expressing functional receptors for Bv8 (Fig. 6), suggesting that GDNF promotes de novo synthesis of PKR1/2 in these cells, in much the same way as GDNF does for bradykinin $\mathrm{B} 1$ receptors in the same population (Vellani et al., 2004). GDNF is released after nerve damage (Trupp et al., 1995; Naveilhan et al., 1997) and may therefore play an important role in upregulating receptors for Bv8, after damage and inflammation, in the nonpeptidergic C-fiber population of neurons.

The present experiments have established that activation of PKRs potentiates gating of TRPV1, via a pathway involving $\mathrm{PKC} \varepsilon$, but this is not necessarily the only mechanism by which PKRs cause hyperalgesia. Injection of Bv8 also causes mechanical hyperalgesia (Negri et al., 2002), an action that has not been addressed by the experiments in the present study. There is evidence that PKC $\varepsilon$ may modulate the threshold of the nociceptorspecific sodium channel, $\mathrm{Na}_{\mathrm{V}} 1.8$ (Khasar et al., 1999), an action that may cause hyperalgesia to mechanical stimuli as well as to heat. PKR activation may also act via signaling pathways other than PKC $\varepsilon$ to cause mechanical hyperalgesia. We note, however, that mechanical hyperalgesia can be caused directly by activation of TRPV1 (Culp et al., 1989; Liu et al., 1998; Walker et al., 2003), and thus it is possible that part or all of the mechanical hyperalgesia after Bv8 injection may result from an action via TRPV1. 
The potency of the actions of $\mathrm{Bv} 8$, both in vivo and in vitro, together with the specific expression of receptors for Bv8 in subpopulations of nociceptive neurons, strongly suggests that PK1 and $\mathrm{PK} 2$, the mammalian homologues of Bv8, play a role in physiological hyperalgesia after injury, infection, and inflammation. The mechanism of release of prokineticins, however, and the circumstances under which they contribute to hyperalgesia, still remain to be established.

\section{References}

Amaya F, Oh-hashi K, Naruse Y, Iijima N, Ueda M, Shimosato G, Tominaga M, Tanaka Y, Tanaka M (2003) Local inflammation increases vanilloid receptor 1 expression within distinct subgroups of DRG neurons. Brain Res 963:190-196.

Bandell M, Story GM, Hwang SW, Viswanath V, Eid SR, Petrus MJ, Earley TJ, Patapoutian A (2004) Noxious cold ion channel TRPA1 is activated by pungent compounds and bradykinin. Neuron 41:849-857.

Caterina MJ, Schumacher MA, Tominaga M, Rosen TA, Levine JD, Julius D (1997) The capsaicin receptor: a heat-activated ion channel in the pain pathway. Nature 389:816-824.

Caterina MJ, Rosen TA, Tominaga M, Brake AJ, Julius D (1999) A capsaicin-receptor homologue with a high threshold for noxious heat. Nature 398:436-441.

Caterina MJ, Leffler A, Malmberg AB, Martin WJ, Trafton J, Petersen-Zeitz KR, Koltzenburg M, Basbaum AI, Julius D (2000) Impaired nociception and pain sensation in mice lacking the capsaicin receptor. Science 288:306-313.

Cesare P, Dekker LV, Sardini A, Parker PJ, McNaughton PA (1999) Specific involvement of PKC-epsilon in sensitization of the neuronal response to painful heat. Neuron 23:617-624.

Copray JC, Mantingh-Otter IJ, Brouwer N (1994) Expression of calciumbinding proteins in the neurotrophin-3-dependent subpopulation of rat embryonic dorsal root ganglion cells in culture. Brain Res Dev Brain Res $81: 57-65$

Culp WJ, Ochoa J, Cline M, Dotson R (1989) Heat and mechanical hyperalgesia induced by capsaicin. Cross modality threshold modulation in human C nociceptors. Brain 112:1317-1331.

Davis JB, Gray J, Gunthorpe MJ, Hatcher JP, Davey PT, Overend P, Harries MH, Latcham J, Clapham C, Atkinson K, Hughes SA, Rance K, Grau E, Harper AJ, Pugh PL, Rogers DC, Bingham S, Randall A, Sheardown SA (2000) Vanilloid receptor-1 is essential for inflammatory thermal hyperalgesia. Nature 405:183-187.

Jordt SE, Bautista DM, Chuang HH, McKemy DD, Zygmunt PM, Hogestatt ED, Meng ID, Julius D (2004) Mustard oils and cannabinoids excite sensory nerve fibres through the TRP channel ANKTM1. Nature 427:260-265.

Khasar SG, Lin Y-H, Martin A, Dadgar J, McMahon T, Wang D, Hundle B, Aley KO, Isenberg W, McCarter G, Green PG, Hodge CW, Levine JD, Messing RO (1999) A novel nociceptor signaling pathway revealed in protein kinase c epsilon mutant mice. Neuron 24:253-260.

LeCouter J, Zlot C, Tejada M, Peale F, Ferrara N (2004) Bv8 and endocrine gland-derived vascular endothelial growth factor stimulate hematopoiesis and hematopoietic cell mobilization. Proc Natl Acad Sci USA 101:16813-16818.

Lee MK, Cleveland DW (1996) Neuronal intermediate filaments. Annu Rev Neurosci 19:187-217.

Li M, Bullock CM, Knauer DJ, Ehlert FJ, Zhou QY (2001) Identification of two prokineticin cDNAs: recombinant proteins potently contract gastrointestinal smooth muscle. Mol Pharmacol 59:692-698.

Lin DC, Bullock CM, Ehlert FJ, Chen JL, Tian H, Zhou QY (2002) Identification and molecular characterization of two closely related $G$ proteincoupled receptors activated by prokineticins/endocrine gland vascular endothelial growth factor. J Biol Chem 277:19276-19280.

Liu M, Max MB, Robinovitz E, Gracely RH, Bennett GJ (1998) The human capsaicin model of allodynia and hyperalgesia: sources of variability and methods for reduction. J Pain Symptom Manage 16:10-20.

Ma QP (2002) Expression of capsaicin receptor (VR1) by myelinated primary afferent neurons in rats. Neurosci Lett 319:87-90.

Martucci C, Franchi S, Giannini E, Tian H, Melchiorri P, Negri L, Sacerdote P (2006) Bv8, the amphibian homologue of the mammalian prokineticins, induces a proinflammatory phenotype of mouse macrophages. Br J Pharmacol 147:225-234.

Masuda Y, Takatsu Y, Terao Y, Kumano S, Ishibashi Y, Suenaga M, Abe M, Fukusumi S, Watanabe T, Shintani Y, Yamada T, Hinuma S, Inatomi N, Ohtaki T, Onda H, Fujino M (2002) Isolation and identification of EGVEGF/prokineticins as cognate ligands for two orphan G-proteincoupled receptors. Biochem Biophys Res Commun 293:396-402.

Mollay C, Wechselberger C, Mignogna G, Negri L, Melchiorri P, Barra D, Kreil G (1999) Bv8, a small protein from frog skin and its homologue from snake venom induce hyperalgesia in rats. Eur J Pharmacol 374:189-196

Naveilhan P, ElShamy WM, Ernfors P (1997) Differential regulation of mRNAs for GDNF and its receptors Ret and GDNFR alpha after sciatic nerve lesion in the mouse. Eur J Neurosci 9:1450-1460.

Negri L, Lattanzi R, Giannini E, Metere A, Colucci M, Barra D, Kreil G, Melchiorri P (2002) Nociceptive sensitization by the secretory protein Bv8. Br J Pharmacol 137:1147-1154.

Numazaki M, Tominaga T, Toyooka H, Tominaga M (2002) Direct phosphorylation of capsaicin receptor VR1 by protein kinase Cepsilon and identification of two target serine residues. J Biol Chem 277:13375-13378.

Rashid MH, Inoue M, Bakoshi S, Ueda H (2003) Increased expression of vanilloid receptor 1 on myelinated primary afferent neurons contributes to the antihyperalgesic effect of capsaicin cream in diabetic neuropathic pain in mice. J Pharmacol Exp Ther 306:709-717.

Schaap D, Parker PJ, Bristol A, Kriz R, Knopf J (1989) Unique substrate specificity and regulatory properties of PKC-epsilon-a rationale for diversity. FEBS Lett 243:351-357.

Schweitz H, Bidard JN, Lazdunski M (1990) Purification and pharmacological characterization of peptide toxins from the black mamba (Dendroaspis polylepis) venom. Toxicon 28:847-856.

Snider WD, McMahon SB (1998) Tackling pain at the source: new ideas about nociceptors. Neuron 20:629-632.

Soga T, Matsumoto S, Oda T, Saito T, Hiyama H, Takasaki J, Kamohara M, Ohishi T, Matsushime H, Furuichi K (2002) Molecular cloning and characterization of prokineticin receptors. Biochim Biophys Acta 1579:173-179.

Tominaga M, Caterina MJ (2004) Thermosensation and pain. J Neurobiol 61:3-12.

Trupp M, Ryden M, Jornvall H, Funakoshi H, Timmusk T, Arenas E, Ibanez CF (1995) Peripheral expression and biological activities of GDNF, a new neurotrophic factor for avian and mammalian peripheral neurons. J Cell Biol 130:137-148.

Vellani V, Mapplebeck S, Moriondo A, Davis JB, McNaughton PA (2001) Protein kinase $\mathrm{C}$ activation potentiates gating of the vanilloid receptor VR1 by capsaicin, protons, heat and anandamide. J Physiol (Lond) 534:813-825.

Vellani V, Zachrisson O, McNaughton PA (2004) Functional bradykinin B1 receptors are expressed in nociceptive neurones and are upregulated by the neurotrophin GDNF. J Physiol (Lond) 560:391-401.

Walker KM, Urban L, Medhurst SJ, Patel S, Panesar M, Fox AJ, McIntyre P (2003) The VR1 antagonist capsazepine reverses mechanical hyperalgesia in models of inflammatory and neuropathic pain. J Pharmacol Exp Ther 304:56-62.

Wechselberger C, Puglisi R, Engel E, Lepperdinger G, Boitani C, Kreil G (1999) The mammalian homologues of frog Bv8 are mainly expressed in spermatocytes. FEBS Lett 462:177-181.

Woodbury CJ, Zwick M, Wang S, Lawson JJ, Caterina MJ, Koltzenburg M, Albers KM, Koerber HR, Davis BM (2004) Nociceptors lacking TRPV1 and TRPV2 have normal heat responses. J Neurosci 24:6410-6415. 